\title{
A Repetição da Valvuloplastia Aórtica por Balão É uma Boa Estratégia para Alívio dos Sintomas e Seleção de Pacientes para Substituição Percutânea da Valva Aórtica
}

\author{
Carla Agatiello1, Deborah Nercolini², Helene Eltchaninoff², Christophe Tron², Alejandro D. Fernandez ${ }^{1}$, \\ José M. Gabay', Carlos Rojas Matas', Daniel Berrocal', Liliana Grinfeld¹, Alain Cribier ${ }^{2}$
}

\begin{abstract}
RESUMO
Introdução: A valvuloplastia aórtica por balão voltou a ganhar interesse desde o início da era da substituição percutânea da valva aórtica, por ser um procedimento que pode ser repetido como ponte e também por ser boa estratégia para a seleção de pacientes para o novo procedimento. Método: De janeiro de 2001 a janeiro de 2009, 174 pacientes consecutivos com estenose aórtica sintomática grave e alto risco cirúrgico calculado pelo EuroSCORE/ STS foram submetidos a valvuloplastia aórtica por balão na França e na Argentina, utilizando-se a mesma técnica. Desse total, $21(12,1 \%)$ precisaram repetir a valvuloplastia aórtica por balão em decorrência de reestenose e os resultados foram comparados aos dos 153 pacientes que realizaram somente o primeiro procedimento. A técnica mais utilizada foi o acesso retrógrado com abordagem femoral utilizando introdutores de $10 \mathrm{~F}, 12 \mathrm{~F}$ ou $14 \mathrm{~F}$, com tamanhos de balão variando de $20 \mathrm{~mm}$ a $23 \mathrm{~mm}$. Resultados: A média de idade foi de $80,4 \pm 9,7$ anos e o EuroSCORE foi de $21 \pm 2 \%$. Abordagem femoral retrógrada foi utilizada em $95 \%$ dos casos. Os resultados do procedimento primário foram os seguintes: a área valvar aórtica aumentou de $0,9 \pm 19 \mathrm{~cm}^{2}$ para $1,02 \pm 0,20 \mathrm{~cm}^{2}$ e o gradiente médio caiu de $50 \pm 21 \mathrm{mmHg}$ para $22 \pm 11 \mathrm{mmHg}$. Os resultados foram semelhantes após a repetição da valvuloplastia aórtica por balão em termos da área valvar aórtica, com melhora de 0,60 $\pm 0,1 \mathrm{~cm}^{2}$ para 1,01 $\pm 0,25 \mathrm{~cm}^{2}$ em um período de $13 \pm 9$ meses entre os dois procedimentos. As taxas de complicações hospitalares foram: morte, $3,3 \%$ vs. $9,5 \% \quad(P=0,20)$; acidente vascular cerebral embólico, $1,3 \%$ vs. $0(P=0,20)$; regurgitação aórtica maciça, $3,3 \%$ vs. $9,5 \%(P=0,20)$; ruptura do balão, $1,3 \%$ vs. 0 $(P=0,20)$; tamponamento pericárdico, 0 vs. $5 \%(P=0,12)$; e complicações vasculares, $3,9 \%$ vs. $9,5 \%(P=0,25)$. No
\end{abstract}

\footnotetext{
1 Hospital Italiano de Buenos Aires - Buenos Aires, Argentina.

${ }^{2}$ Hospital Universitario Charles Nicolle - Rouen, França.

Correspondência: Carla Romina Agatiello. Interventional Cardiology Hospital Italiano de Buenos Aires - Gascon 450 PB 1181 - Buenos Aires, Argentina.

E-mail: carla.agatiello@hospitalitaliano.org.ar, cragatiello@hotmail.com Recebido em: 30/10/2009 • Aceito em: 17/11/2009
}

\section{ABSTRACT}

Repeat Balloon Aortic Valvuloplasty is a Good Strategy for Symptom Relief and Patient Selection for Percutaneous Aortic Valve Replacement

Background: Balloon aortic valvuloplasty (BAV) has regained interest since the onset of percutaneous aortic valve replacement (PAVR) as it can be repeated as a bridge to transcatheter aortic valve implantation (TAVI) and is also a good strategy for patient selection. Method: From January 2001 to January 2009, 174 consecutive patients with severe symptomatic aortic stenosis (AS) and high surgical risk calculated by EuroSCORE/STS underwent BAV in France and Argentina using the same technique. Twenty one (12.1\%) of these patients underwent repeat BAV for restenosis and the results were compared with those of the 153 patients undergoing the primary procedure. The most used technique was retrograde access by femoral approach, using 10, 12 or $14 \mathrm{~F}$ introducers, with balloons sizes ranging from 20 to $23 \mathrm{~mm}$. Results: Patients' mean age was $80.4 \pm 9.7$ years and EuroSCORE was $21 \pm 2 \%$. The retrograde femoral approach was used in $95 \%$ of the cases. Results of the primary procedure were as follows: aortic valve area (AVA) increased from $0.9 \pm 19 \mathrm{~cm}^{2}$ to $1.02 \pm 0.20 \mathrm{~cm}^{2}$ and mean gradient decreased from $50 \pm 21 \mathrm{mmHg}$ to $22 \pm 11 \mathrm{mmHg}$. The results were similar after repeat $\mathrm{BAV}$ in terms of $\mathrm{AVA}$, with an improvement of $0.60 \pm 0.1 \mathrm{~cm}^{2}$ to $1.01 \pm 0.25 \mathrm{~cm}^{2}$ in a period of $13 \pm 9$ months between the two procedures. The rates of complications were: death, $3.3 \%$ vs. $9.5 \%$ $(P=0.20)$; embolic stroke, $1.3 \%$ vs. $0(P=0.20)$; massive aortic regurgitation, $3.3 \%$ vs. $9.5 \%(P=0.20)$; balloon burst, $1.3 \%$ vs. $0(\mathrm{P}=0.20)$; pericardial tamponade, 0 vs. $5 \%(P=0.12)$; and vascular complications, $3.9 \%$ vs. $0.5 \%$ $(P=0.25)$. Forty eight patients $(27.5 \%)$ were selected as 
Agatiello C, et al. A Repetição da Valvuloplastia Aórtica por Balão É uma Boa Estratégia para Alívio dos Sintomas e Seleção de Pacientes para Substituição Percutânea da Valva Aórtica. Rev Bras Cardiol Invas. 2009;17(4):463-9.

total, 48 pacientes $(27,5 \%)$ foram selecionados como bons candidatos para substituição percutânea da valva aórtica durante o período de acompanhamento nos dois países, dos quais 36 realizaram o procedimento na França e 4, na Argentina. Conclusões: A repetição da valvuloplastia aórtica por balão é uma estratégia útil em pacientes idosos nãocirúrgicos com estenose aórtica sintomática grave para aliviar os sintomas após reestenose e como ponte para a substituição percutânea da valva aórtica. Essa técnica pode ser repetida com baixa taxa de complicações.

DESCRITORES: Estenose da valva aórtica/cirurgia. Valva aórtica. Dilatação com balão. Implante de prótese de valva.

$\mathbf{N}$ os últimos anos, a valvuloplastia aórtica por balão voltou a ser utilizada como método de tratamento em pacientes adultos com estenose aórtica grave, graças ao início da era da substituição percutânea da valva aórtica. Embora a técnica da valvuloplastia aórtica por balão tenha tido início promissor para tratar pacientes não-cirúrgicos com estenose aórtica grave, logo começaram a surgir problemas, como morbidade e mortalidade relacionadas ao procedimento e reestenose aórtica ${ }^{1}$. Apesar disso, essa técnica continuou a ser utilizada por seus defensores, que foram introduzindo melhoras no procedimento ${ }^{2-5}$.

$\mathrm{Na}$ atualidade, o envelhecimento da população gera problemas, com o aumento da incidência de doença da valva aórtica e a prevalência de estenose aórtica grave de 2,4\% em indivíduos com 75 a 85 anos de idade e de $4 \%$ em indivíduos com mais de 85 anos $^{6}$.

Embora a terapia de escolha para estenose aórtica sintomática grave seja a cirurgia de substituição da valva aórtica, em muitos casos as comorbidades associadas fazem com que $30 \%$ a $40 \%$ da população afetada não seja qualificada para cirurgia ${ }^{7}$. Levando-se em conta essa população de alto risco cirúrgico, foi desenvolvida a técnica de substituição percutânea da valva aórtica, utilizada pela primeira vez em humanos em $2002^{8}$.

Neste artigo, é discutida a seleção de pacientes com estenose aórtica sintomática grave para a substituição percutânea da valva aórtica e o papel da valvuloplastia aórtica por balão de repetição como uma ponte para a terapia definitiva.

\section{MÉTODO}

Entre janeiro de 2001 e janeiro de 2009, os pacientes com estenose aórtica sintomática grave e alto risco cirúrgico, de acordo com o EuroSCORE/STS ${ }^{9-10}$, submetidos a valvuloplastia aórtica com balão foram avaliados no Charles Nicolle Hospital (2002-2005, Rouen, França) e no Hospital Italiano de Buenos Aires (2006-2009, Buenos Aires, Argentina). good candidates for PAVR during the follow up period in both countries. Thirty six patients underwent PAVR in France and four patients in Argentina. Conclusions: Repeat BAV is a useful strategy in nonsurgical elderly patients with severe symptomatic AS to relieve symptoms after restenosis and as a bridge to transcatheter aortic valve implantation. This technique can be repeated with low complication rates.

DESCRIPTORS: Aortic valve stenosis/surgery. Aortic valve. Balloon dilatation. Heart valve prosthesis implantation.

Todos os pacientes realizaram valvuloplastia aórtica por balão como recurso paliativo para melhorar os sintomas e otimizar a seleção de pacientes para substituição percutânea da valva aórtica como indicação IIB das diretrizes do American College of Cardiology/ American Heart Association (ACC/AHA) ${ }^{11}$.

A técnica para valvuloplastia aórtica por balão e valvuloplastia aórtica por balão de repetição foi realizada principalmente pelo acesso retrógrado, utilizando $70 \mathrm{U} / \mathrm{kg}$ de heparina no decorrer do procedimento. Durante a insuflação do balão, foi realizada estimulação rápida com marca-passo transitório (180/220 batidas/ minuto), suficiente para permitir a redução das pressões intracavitárias e evitar a movimentação do balão. Essa técnica foi desenvolvida por Cribier e posteriormente aperfeiçoada por Webb'12 (Figura 1).

Em todos os casos, foi realizada ecocardiografia Doppler transtorácica ou transesofágica para avaliar o diâmetro do anel aórtico e ajustar a medida do balão a ser utilizado. Nos dois países foram utilizados baIões de $20 \mathrm{~mm}$ a $23 \mathrm{~mm}$ (NuMed Inc. - Hopkinton, Estados Unidos, e BALT - Montmorency, França), utilizando-se introdutor de $10 \mathrm{~F}$ a $14 \mathrm{~F}$. Em alguns casos, foram utilizados balões de $18 \mathrm{~mm}$ ou $25 \mathrm{~mm}$.

Os candidatos a valvuloplastia aórtica por balão ou valvuloplastia aórtica por balão de repetição foram pacientes com estenose aórtica grave avaliados no laboratório de cateterização por termodiluição, com área valvar aórtica $<1 \mathrm{~cm}^{2}$ ou gradiente transvalvar aórtico > $25 \mathrm{mmHg}$ e insuficiência aórtica leve a moderada, comprovada por aortografia em projeção oblíqua anterior esquerda a 30 graus.

Para avaliar a viabilidade da substituição percutânea da valva aórtica e o tamanho da bioprótese aórtica a ser implantada, foi realizada ecocardiografia transesofágica em toda a população com indicação para essa técnica, tendo sido anotada a medida do balão utilizado durante a valvuloplastia aórtica por balão (Figura 2).

Os pacientes que realizaram valvuloplastia aórtica por balão na França foram avaliados para as biopróteses 
Agatiello C, et al. A Repetição da Valvuloplastia Aórtica por Balão É uma Boa Estratégia para Alívio dos Sintomas e Seleção de Pacientes para Substituição Percutânea da Valva Aórtica. Rev Bras Cardiol Invas. 2009;17(4):463-9.

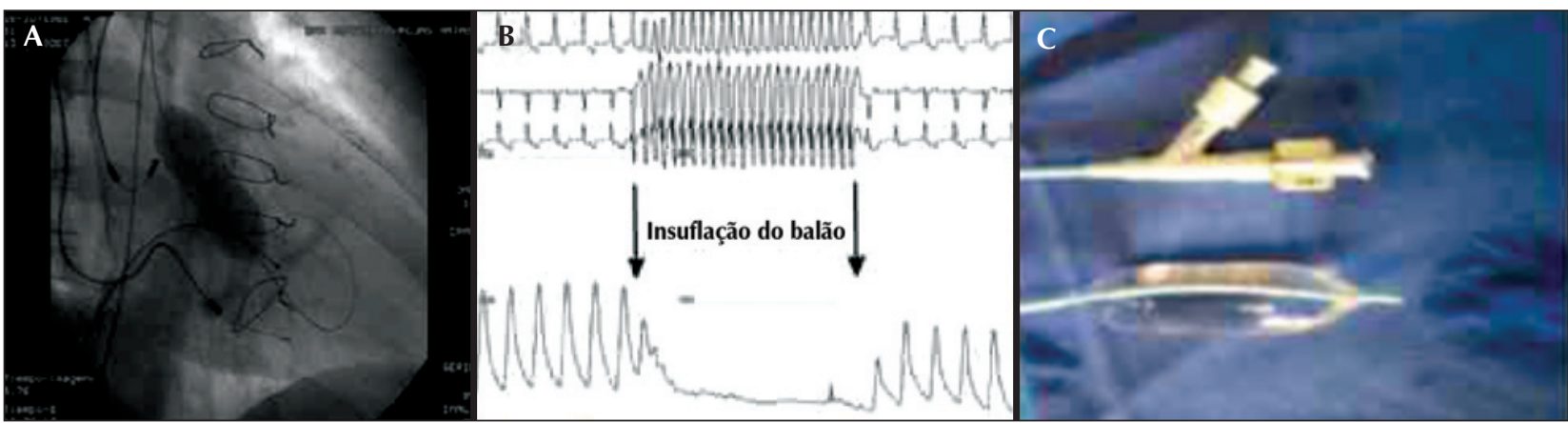

Figura 1 - Estimulação rápida no momento da insuflação do balão. Em A e B, observa-se o balão imóvel na porção média da valva aórtica, favorecido por queda de pressão arterial durante estimulação rápida Em C, balão Cristal (BALT).
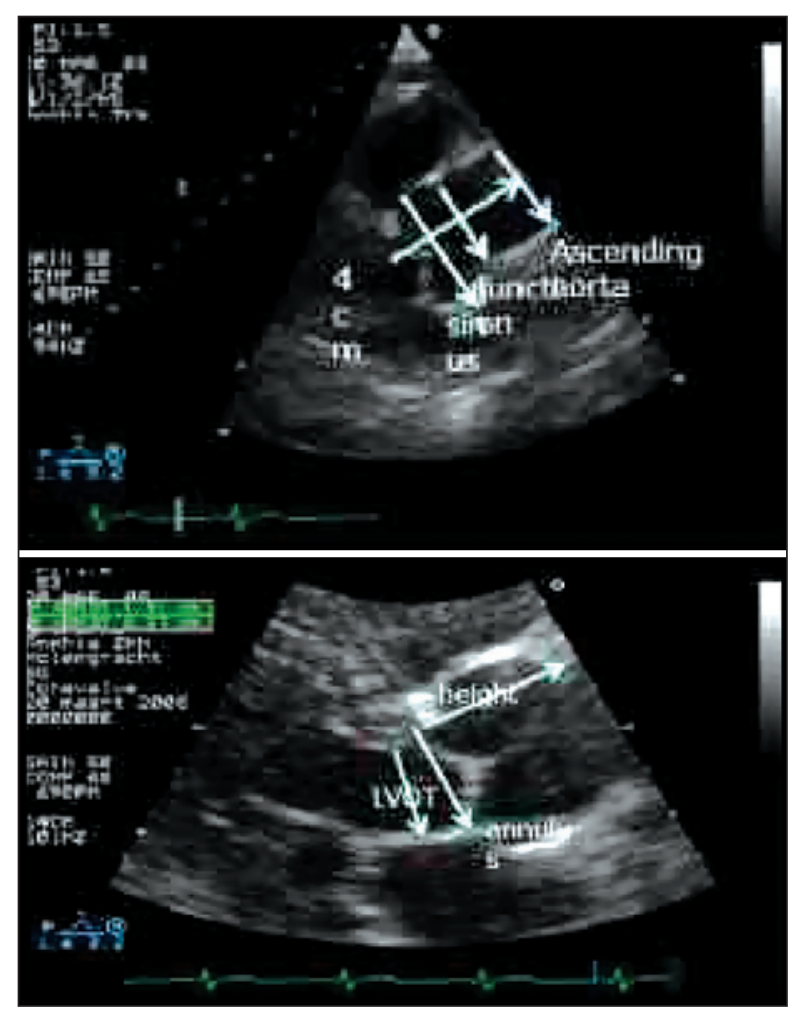

Figura 2 - Visão do eixo longo paraesternal. As medidas foram realizadas durante ecocardiografia Doppler transesofágica para avaliar o tamanho do balão e o tamanho da bioprótese aórtica.

aórticas Cribier-Edwards ${ }^{\text {TM }}$ e Edwards-SAPIEN ${ }^{\text {TM }}$ (Edwards Lifesciences - Irvine, Estados Unidos) entre 2002 e 2005, como parte dos estudos Initial Registry of Endovascular Implantation of Valves in Europe (I-REVIVE) e Registry of Endovascular Critical Aortic Stenosis Treatment (RECAST). Os pacientes que realizaram valvuloplastia aórtica por balão na Argentina foram avaliados para a bioprótese aórtica Edwards-SAPIEN ${ }^{\mathrm{TM}}$ e, posteriormente, para a CoreValve $^{T M}$ (Medtronic - Minneapolis, Estados Unidos).

Todos os pacientes assinaram o termo de consentimento informado para "Valvuloplastia Aórtica por
Balão", na presença de uma testemunha. Para os pacientes em choque ou com diagnóstico de demência senil, o consentimento foi obtido por meio de um tutor ou representante legal.

As variáveis contínuas foram expressas como média e desvio padrão e comparadas com teste $t$ de Student. Os dados categóricos foram expressos em números e porcentagens e comparados com o teste de qui-quadrado ou o teste exato de Fisher, quando indicado. Valor de $\mathrm{P}<0,05$ foi considerado estatisticamente significante.

\section{RESULTADOS}

No total, foram avaliados 174 pacientes com estenose aórtica sintomática grave submetidos a valvuloplastia aórtica por balão. A média de idade foi de 80,4 $\pm 9,7$ anos e o EuroSCORE Logístico, de $21 \pm 2 \%$. Desses pacientes, $21(12,1 \%)$ realizaram valvuloplastia aórtica por balão de repetição em decorrência de reestenose, como uma ponte para a substituição percutânea da valva aórtica, e seus resultados foram comparados aos dos 153 pacientes que realizaram apenas a primeira valvuloplastia aórtica por balão. As características dessa população estão apresentadas na Tabela 1.

Não houve diferenças significativas entre as características clínicas dos pacientes que realizaram uma ou mais valvuloplastias, exceto a classe funcional III-IV da NYHA, mais frequente para o grupo com valvuloplastia aórtica por balão $(89,5 \%$ vs. 71,4\%; $\mathrm{P}=0,03)$, e o EuroSCORE Logístico, mais alto para o grupo com valvuloplastia aórtica por balão de repetição (21 $\pm 2 \%$ vs. $23 \pm 3 \% ; P<0,001)$.

Em relação aos resultados da valvuloplastia aórtica por balão, houve redução do gradiente aórtico transvalvar de $50 \pm 21 \mathrm{mmHg}$ para $22 \pm 11 \mathrm{mmHg}$ e melhora da área valvar aórtica de 0,59 $\pm 0,19 \mathrm{~cm}^{2}$ para 1,02 $\pm 0,20 \mathrm{~cm}^{2}$. Os mesmos resultados foram obtidos após valvuloplastia aórtica por balão de repetição, $13 \pm 9$ meses após o procedimento inicial, com melhora da área valvar aórtica de 0,60 $\pm 0,10 \mathrm{~cm}^{2}$ para 1,01 $\pm 0,25 \mathrm{~cm}^{2}$. 
Agatiello C, et al. A Repetição da Valvuloplastia Aórtica por Balão É uma Boa Estratégia para Alívio dos Sintomas e Seleção de Pacientes para Substituição Percutânea da Valva Aórtica. Rev Bras Cardiol Invas. 2009;17(4):463-9.

As complicações hospitalares estão apresentadas na Tabela 2. Para os pacientes submetidos a valvuloplastia aórtica por balão e valvuloplastia aórtica por balão de repetição, houve, respectivamente, óbito em $3,3 \%$ vs. $9,5 \%(P=0,20)$, acidente vascular cerebral embólico em 1,3\% vs. 0 ( $P>0,99)$, regurgitação aórtica maciça em $3,3 \%$ vs. $9,5 \%(P=0,20)$, ruptura do balão em $1,3 \%$ vs. 0 ( $\mathrm{P}>0,99)$, tamponamento pericárdico em 0 vs. $5 \%(P=0,12)$ e complicações vasculares em $3,9 \%$ vs. $9,5 \%(P=0,25)$.

Dos 174 pacientes que realizaram valvuloplastia aórtica por balão e valvuloplastia aórtica por balão de repetição, 40 (23\%) foram selecionados como bons candidatos para substituição percutânea da valva aórtica (Figura 3).
Nos dois países, EuroSCORE Logístico > 15\%, diâmetro do anel aórtico de $18 \mathrm{~mm}$ a $25 \mathrm{~mm}$ para Edwards-SAPIEN ${ }^{\mathrm{TM}}$ ou de $20 \mathrm{~mm}$ a $27 \mathrm{~mm}$ para CoreValve $^{T M}$, e diâmetro dos vasos periféricos $>8 \mathrm{~mm}$ para a bioprótese Edwards-SAPIEN ${ }^{\mathrm{TM}}$ e $>6 \mathrm{~mm}$ para a CoreValve ${ }^{\mathrm{TM}}$ foram considerados para definir a elegibilidade da substituição percutânea da valva aórtica.

Entre os pacientes selecionados, 36 receberam implante da bioprótese Cribier-Edwards/Edwards-SAPIEN ${ }^{\text {TM }}$, na França, e 4 pacientes receberam a bioprótese CoreValve ${ }^{T M}$, na Argentina.

\section{DISCUSSÃO}

Conforme observado na população analisada, os pacientes com estenose aórtica grave e alto risco para

Tabela 1

Características da população

\begin{tabular}{lccc}
\hline & $\begin{array}{c}\text { VAB } \\
\mathbf{2 0 0 2 - 2 0 0 9} \\
(\mathbf{n}=\mathbf{1 5 3})\end{array}$ & $\begin{array}{c}\text { Re-VAB } \\
\mathbf{2 0 0 2 - 2 0 0 9} \\
(\mathbf{n = 2 1 )}\end{array}$ & P \\
\hline Idade, anos & $80,4 \pm 9,7$ & $83,4 \pm 7,0$ & 0,17 \\
EuroSCORE, $\%$ & $21 \pm 2$ & $23 \pm 3$ & $<0,001$ \\
Sexo feminino & $72(47,1 \%)$ & $13(61,9 \%)$ & 0,25 \\
Comorbidades $>3$ & $113(73,8 \%)$ & $15(71,4 \%)$ & 0,79 \\
História de câncer & $30(19,6 \%)$ & $1(4,8 \%)$ & 0,13 \\
História de AVC & $15(9,8 \%)$ & $2(9,5 \%)$ & $>0,99$ \\
História de CRM & $27(17,6 \%)$ & $4(19 \%)$ & $>0,99$ \\
Insuficiência renal & $30(19,6 \%)$ & $6(28,5 \%)$ & 0,39 \\
Classe III-IV da NYHA & $137(89,5 \%)$ & $15(71,4 \%)$ & 0,03 \\
VAB de emergência & $14(9,1 \%)$ & $1(4,8 \%)$ & $>0,99$ \\
\hline
\end{tabular}

$\mathrm{AVC}=$ acidente vascular cerebral; $\mathrm{CRM}=$ cirurgia de revascularização miocárdica; $\mathrm{n}=$ número de pacientes; $\mathrm{NYHA}=\mathrm{New}$ York Heart Association; Re-VAB = valvuloplastia aórtica por balão de repetição; VAB = valvuloplastia aórtica por balão.

Tabela 2

Complicações observadas entre valvuloplastia aórtica por balão e valvuloplastia aórtica por balão de repetição

\begin{tabular}{|c|c|c|c|}
\hline & $\begin{array}{c}\text { VAB } \\
2002-2009 \\
(n=153)\end{array}$ & $\begin{array}{c}\text { Re-VAB } \\
2002-2009 \\
(n=21)\end{array}$ & $\mathbf{P}$ \\
\hline Mortes hospitalares & $5(3,3 \%)$ & $2(9,5 \%)$ & 0,20 \\
\hline Mortes periprocedimento & $2(1,3 \%)$ & $2(9,5 \%)$ & 0,07 \\
\hline AVC & $2(1,3 \%)$ & 0 & $>0,99$ \\
\hline Arritmias não-fatais & $5(3,3 \%)$ & 0 & $>0,99$ \\
\hline Ruptura do balão & $2(1,3 \%)$ & 0 & $>0,99$ \\
\hline Tamponamento cardíaco & 0 & $1(5 \%)$ & 0,12 \\
\hline Complicações vasculares & $6(3,9 \%)$ & $2(9,5 \%)$ & 0,25 \\
\hline
\end{tabular}


Agatiello C, et al. A Repetição da Valvuloplastia Aórtica por Balão É uma Boa Estratégia para Alívio dos Sintomas e Seleção de Pacientes para Substituição Percutânea da Valva Aórtica. Rev Bras Cardiol Invas. 2009;17(4):463-9.

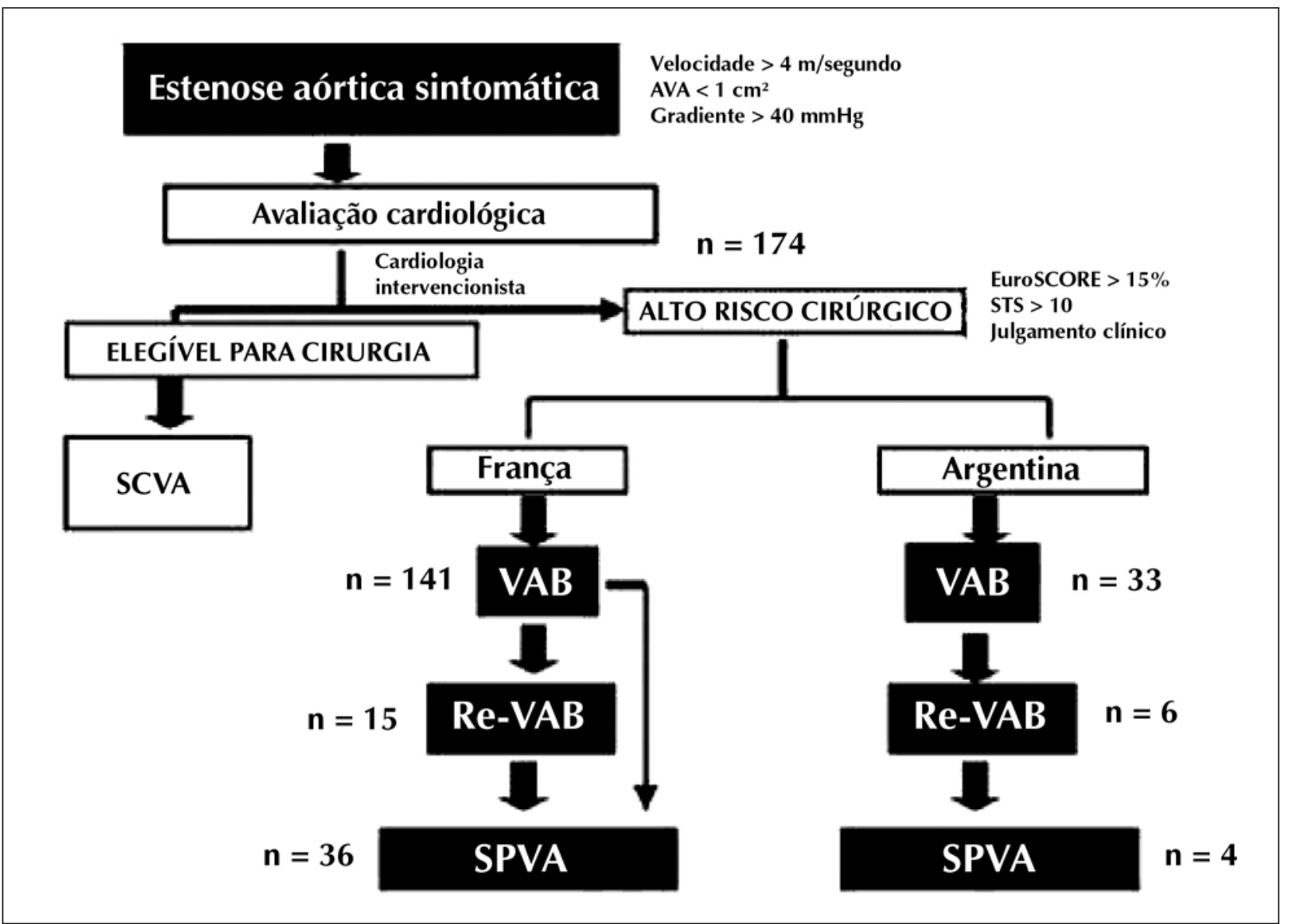

Figura 3 - Fluxo de pacientes do estudo. AVA = área valvar aórtica; $n$ = número de pacientes; Re-VAB = valvuloplastia aórtica por balão de repetição; SCVA = substituição cirúrgica da valva aórtica; SPVA = substituição percutânea da valva aórtica; VAB = valvuloplastia aórtica por balão.

cirurgia cardíaca continuam sendo um problema médico devido ao envelhecimento da população, semeIhante na Europa e na América Latina. Apenas uma parte dessa população é tratada com a cirurgia de substituição da valva aórtica, e $30 \%$ a $44 \%$ não recebem tratamento $^{(7)}$.

Para os pacientes que não podem realizar a cirurgia, a valvuloplastia aórtica por balão foi desenvolvida como método paliativo ${ }^{1,11}$, que atualmente corresponde a uma indicação de classe IIB. Apesar das limitações conhecidas $(80 \%$ de reestenose após um ano), esse procedimento atualmente tem baixa taxa de complicações ao se comparar o Registro Mansfield ${ }^{3}$ às séries do Hospital Universitário de Rouen ${ }^{4}$ e de Agarwal et al. ${ }^{13}$. $\mathrm{Na}$ era da substituição percutânea da valva aórtica, observou-se o renascimento da valvuloplastia aórtica por balão ${ }^{8}$ e houve aumento da necessidade de familiarizar diferentes equipes médicas com essa técnica, que estava praticamente esquecida ${ }^{14-18}$.

Os avanços tecnológicos melhoraram os resultados da valvuloplastia aórtica por balão e reduziram suas complicações, como a disponibilidade dos dispositivos de fechamento vascular percutâneo (Angioseal ${ }^{\mathrm{TM}}$,
St. Jude Medical - St. Paul, Estados Unidos; e Prostar ${ }^{\mathrm{TM}}$, Abbott Vascular Devices - Santa Clara, Estados Unidos $)^{5}$. As complicações vasculares têm diminuído ao longo do tempo, de 10\% no Registro Mansfield ${ }^{3}$ para menos de $5 \%$ na série do Hospital Universitário de Rouen ${ }^{4}$. Adicionalmente, o uso de estimulação rápida, descrito por Webb et al. ${ }^{12}$, reduziu as perfurações ventriculares e a incidência de acidente vascular cerebral.

Na população do Hospital Universitário de Rouen, 21 pacientes foram submetidos a dois procedimentos de valvuloplastia aórtica por balão (12\%) antes de serem considerados candidatos a substituição percutânea da valva aórtica, em decorrência do início recente da nova técnica nos dois países e à acurada seleção de casos. Todos os pacientes que realizaram valvuloplastia aórtica por balão e valvuloplastia aórtica por balão de repetição foram avaliados por aortografia abdominal para verificar o diâmetro dos acessos vasculares periféricos (mínimo de $8 \mathrm{~mm}$ para a bioprótese EdwardsSAPIEN $^{\text {TM }}$ e de $6 \mathrm{~mm}$ para a bioprótese CoreValve ${ }^{\mathrm{TM}}$ ) e por angiotomografia da aorta torácica e abdominal.

Na série do Hospital Universitário de Rouen, os pacientes realizaram nova valvuloplastia aórtica por 
Agatiello C, et al. A Repetição da Valvuloplastia Aórtica por Balão É uma Boa Estratégia para Alívio dos Sintomas e Seleção de Pacientes para Substituição Percutânea da Valva Aórtica. Rev Bras Cardiol Invas. 2009;17(4):463-9.

balão em decorrência de reestenose aórtica sintomática e para reavaliar o tamanho do anel aórtico por meio do balão insuflado. A maioria dos pacientes com anéis aórticos pequenos (entre $17 \mathrm{~mm}$ e $20 \mathrm{~mm}$ ) foi submetida a valvuloplastia aórtica com balão de $20 \mathrm{~mm}$ (balão NuMED) para avaliar a colocação de bioprótese CoreValve ${ }^{\mathrm{TM}}$ (na Argentina). Pacientes com anéis aórticos grandes (> $25 \mathrm{~mm}$ ) foram submetidos a valvuloplastia aórtica com balão de $25 \mathrm{~mm}$ para avaliar a colocação da bioprótese de Edwards-SAPIEN ${ }^{\mathrm{TM}}$ (na França), tamanho limite exigido para esse dispositivo.

Embora já tenha sido descrito que a valvuloplastia aórtica por balão não altera a evolução natural da doença aórtica, Agarwal et al. descreveram que os pacientes que realizaram valvuloplastia aórtica por balão de repetição apresentaram melhora das curvas de sobrevivência após um ano, permitindo que maior número de pacientes fizesse ponte para um tratamento mais definitivo ${ }^{13}$.

O objetivo desta análise retrospectiva não foi comparar a valvuloplastia aórtica por balão com a substituição percutânea da valva aórtica. Para que isso ocorra, será preciso aguardar os resultados do estudo randomizado Placement of Aortic Transcatheter Valve (PARTNER), que compara o tratamento médico, a substituição percutânea da valva aórtica e a substituição cirúrgica da valva aórtica ${ }^{18}$. Não se tentou comparar os resultados das biopróteses implantadas, embora em geral se possa dizer que ambas demonstraram melhora significativa do gradiente e da área valvar após o implante. É importante destacar que apenas $23 \%$ dos pacientes avaliados eram qualificados para substituição percutânea da valva aórtica; portanto, a valvuloplastia aórtica por balão ainda é uma importante ferramenta e uma condição necessária ao se planejar o início de um programa de substituição percutânea da valva aórtica.

Nas séries avaliadas, não ocorreu evento cardiovascular maior nos pacientes submetidos a valvuloplastia aórtica por balão de repetição. Argawal et al. descreveram que, de acordo com sua experiência, o período sem sintomas entre a primeira e a segunda valvuloplastias aórticas por balão foi de $18 \pm 3$ meses, e que entre a segunda e a terceira valvuloplastias aórticas por balão esse período foi de $15 \pm 4$ meses $^{13}$. Não há relatos na literatura de casos com mais de três valvuloplastias aórticas por balão, fato que poderia ser explicado pela idade avançada dessa população e também por resultados insatisfatórios. Vale mencionar que quanto menor o gradiente final observado $(<15 \mathrm{mmHg})$ melhor será o resultado clínico e menor a necessidade de se repetir a valvuloplastia aórtica por balão.

Feldman et al. não apoiam a estratégia de valvuloplastia aórtica por balão de repetição, pois observaram aumento insignificante da área valvar após segunda valvuloplastia aórtica por balão, que atribuíram à "ossificação da valva aórtica na ocasião da reestenose"16.

\section{CONCLUSÕES}

A valvuloplastia aórtica por balão de repetição é uma estratégia útil no tratamento de pacientes de alto risco, com estenose aórtica sintomática grave, para aliviar os sintomas após reestenose da valva aórtica. Essa técnica ajuda na seleção de casos qualificados para substituição percutânea da valva aórtica, assim como faz uma ponte para substituição percutânea da valva aórtica, com baixa incidência de complicações.

\section{CONFLITO DE INTERESSES}

Alain Cribier é consultor da Edwards-Lifesciences. Os demais autores declararam inexistência de conflito de interesses.

\section{REFERÊNCIAS BIBLIOGRÁFICAS}

1. Cribier A, Savin T, Saoudi N, Rocha P, Berland J, Letac B. Percutaneous transluminal valvuloplasty of acquired aortic stenosis in elderly patients: an alternative to valve replacement? Lancet. 1986;1(8472):63-7.

2. Eltchaninoff $\mathrm{H}$, Cribier A, Tron C, Anselme F, Koning R, Soyer R, et al. Balloon aortic valvuloplasty in elderly patients at high risk for surgery, or inoperable. Immediate and midterm results. Eur Heart J. 1995;16(8):1079-84.

3. McKay R. The Mansfield Scientific Aortic Valvuloplasty Registry: overview of acute hemodynamic results and procedural complications. Am Coll Cardiol. 1991;17(2):485-91.

4. Agatiello $\mathrm{C}$, Eltchaninoff $\mathrm{H}$, Tron $\mathrm{C}$, Bauer $\mathrm{F}$, Babaliaros $\mathrm{V}$, Nercolini D, et al. [Balloon aortic valvuloplasty in the adult. Immediate results and in-hospital complications in the latest series of 141 consecutive patients at the University Hospital of Rouen (2002-2005)]. Arch Mal Coeur Vaiss. 2006; 99(3):195-200

5. Solomon LW, Fusman B, Jolly N, Kim A, Feldman T. Percutaneous suture closure for management of large French size arterial puncture in aortic valvuloplasty. J Invasive Cardiol. 2001;13(8):592-6.

6. Carabello BA, Paulus WJ. Aortic stenosis. Lancet. 2009; 373(9667):956-66. Comment in: Lancet. 2009;373(9680): 2022-3; author reply 2023, Lancet. 2009;373(9680):2023; author reply 2023.

7. lung B, Baron G, Butchart EG, Delahaye F, Gohlke-Bärwolf C, Levang OW, et al. A prospective survey of patients with valvular heart disease in Europe: The Euro Heart Survey on Valvular Heart Disease. Eur Heart J. 2003;24(13):1231-43.

8. Cribier A, Eltchaninoff H, Tron C, Bauer F, Agatiello C, Sebagh L, et al. Early experience with percutaneous transcatheter implantation of heart valve prosthesis for the treatment of end-stage inoperable patients with calcific aortic stenosis. J Am Coll Cardiol. 2004;43(4):698-703.

9. Roques F, Nashef SA, Michel P, Gauducheau E, de Vincentiis C, Baudet E, et al. Risk factors and outcome in European cardiac surgery: analysis of the EuroSCORE multinational database of 19030 patients. Eur J Cardiothorac Surg. 1999;15(6): 816-22; discussion 822-3.

10. Wendt D, Osswald BR, Kayser K, Thielmann M, Tossios P, Massoudy $\mathrm{P}$, et al. Society of Thoracic Surgeons score is superior to the EuroSCORE determining mortality in high risk patients undergoing isolated aortic valve replacement. Ann Thorac Surg. 2009;88(2):468-4; discussion 474-5.

11. Bonow RO, Carabello BA, Chatterjee K, de Leon AC Jr, Faxon DP, Freed MD, Gaasch WH, Lytle BW, Nishimura RA, 
Agatiello C, et al. A Repetição da Valvuloplastia Aórtica por Balão É uma Boa Estratégia para Alívio dos Sintomas e Seleção de Pacientes para Substituição Percutânea da Valva Aórtica. Rev Bras Cardiol Invas. 2009;17(4):463-9.

O'Gara PT, O'Rourke RA, Otto CM, Shah PM, Shanewise JS; American College of Cardiology/American Heart Association Task Force on Practices Guidelines. 2008 focused update incorporated into the ACC/AHA 2006 guidelines for the management of patients with valvular heart disease: a report of the American College of Cardiology/American Heart Association Task Force on Practice Guidelines (Writing Committee to revise the 1998 guidelines for the management of patients with valvular heart disease). Endorsed by the Society of Cardiovascular Anesthesiologists, Society for Cardiovascular Angiography and Interventions, and Society of Thoracic Surgeons. J Am Coll Cardiol. 2008;52(13):e1-e142.

12. Webb JG, Pasupati S, Achtem L, Thompson CR. Rapid pacing to facilitate transcatheter prosthetic heart valve implantation. Catheter Cardiovasc Interv. 2006;68(2):199-204.

13. Agarwal A, Kini AS, Attanti S, Lee PC, Steinheimer AM, Moreno PR, et al. Results of repeat balloon valvuloplasty for treatment of aortic stenosis in patients aged 59 to 104 years. Am J Cardiol. 2005;95(1):43-7.
14. Koning R, Cribier A, Asselin C, Mouton-Schleifer D, Derumeaux G, Letac B. Repeat balloon aortic valvuloplasty. Cathet Cardiovasc Diagn. 1992;26(4):249-54.

15. Ferguson J. The efficacy of multiple balloon aortic valvuloplasty procedures [abstract]. Circulation. 1988;78(suppl II):II-531.

16. Feldman T, Glagov S, Carroll JD. Restenosis following successful balloon valvuloplasty: bone formation in aortic valve leaflets. Cathet Cardiovasc Diagn. 1993;29(1):1-7.

17. Kuntz RE, Tosteson AN, Berman AD, Goldman L, Gordon PC, Leonarrd BM, et al. Predictors of event free survival after balloon aortic valvuloplasty. N Engl J Med. 1991;325(1):1723. Comment in: N Engl J Med. 1992;326(2):138-9.

18. ClinicalTrials.gov [Internet]. Bethesda (MD): National Library of Medicine (US). 2000 Feb 29. Identifier NCT00530894, The PARTNER trial: placement of aortic transcatheter valve trial; 2007 Sep 14 [updated 2008 Nov 19; cited 2009 Nov 19]; [about 4 screens]. Available from: http://clinicaltrials.gov/ ct2/show/NCT00530894 